\title{
Erratum: Determination of the Thermal Boundary Resistance in the Transport Approach*
}

\author{
N. Perrin \\ Groupe de Physique des Solides de l'Ecole Normale Supérieure, Paris, France
}

(Received June 9, 1978)

On p. 262 , following Eq. (13), $J_{e}=-K_{e} \cdot \nabla T_{e}$, there should appear: "with

$$
-\nabla \cdot J_{e}=\sum_{\mathbf{q}} \hbar \omega\left[\bar{N}\left(T_{e}\right)-N\right] / \tau
$$

The above electron energy balance equation relates the electron temperature to the phonon distribution."

Therefore, on p. 263, in Section 4, the sentence, "The result is injected in Eq. (13) ... ." should be replaced by "The result is injected in Eqs. (13) and (13a) ..."

*This paper appeared in J. Low Temp. Phys. 31 (1/2), 257 (1978). 